05

\title{
Влияние импульсного тока на эффект Портевена-Ле Шателье в алюминий-магниевом сплаве АMг5
}

\author{
() А.А. Шибков, М.Ф. Гасанов, А.А. Денисов, А.Е. Золотов, В.И. Иволгин \\ Тамбовский государственный университет имени Г.Р. Державина, \\ 392000 Тамбов, Россия \\ e-mail: Shibkov@tsu.tmb.ru
}

(Поступило в Редакцию 14 июня 2016 г.)

Проведены экспериментальные исследования влияния импульсов тока на полосообразование и прерывистую деформацию сплава АМг5. Установлено, что для подавления зарождения полосы деформации необходима предварительная обработка сплава током плотностью $60 \mathrm{~A} / \mathrm{mm}^{2}$ в течение не менее $1 \mathrm{~s}$.

DOI: 10.21883/JTF.2017.04.44329.1994

Как известно, электропластический эффект (ЭПЭ) состоит в резком падении напряжения течения при пропускании через деформируемый образец коротких (длительностью $\sim 30-100 \mu \mathrm{s}$ ) импульсов тока большой плотности $\sim 10^{3}-10^{4} \mathrm{~A} / \mathrm{mm}^{2}[1,2]$. После окончания импульса тока напряжение течения восстанавливается до исходного значения. При пропускании последовательности импульсов тока кривая деформации металлического сплава приобретает скачкообразный характер [1-3]. В настоящее время ЭПЭ обнаружен и исследован у многих металлов и сплавов, которые в отсутствие импульсов тока демонстрируют гладкие (без скачков) кривые деформации [3]. Авторы настоящей работы обнаружили феноменологически „обратный“ электропластическому эффект, состоящий в подавлении постоянным электрическим током небольшой плотности $10-60 \mathrm{~A} / \mathrm{mm}^{2}$ скачкообразной (прерывистой) деформации Портевена-Ле Шателье (ПЛШ) с одновременным увеличением напряжения течения в некоторых авиационных сплавах систем $\mathrm{Al}-\mathrm{Mg}-\mathrm{Mn}, \mathrm{Al}-\mathrm{Li}-\mathrm{Mg}$ и $\mathrm{Al}-\mathrm{Zn}-\mathrm{Cu}-\mathrm{Mg}$ [4-6], которые в отсутствие тока демонстрируют явно выраженный эффект ПЛШ при комнатной и повышенных температурах. Явление подавления постоянным током прерывистой деформации подтверждено на алюминий-магниевом сплаве АМг3 (АА 5754-0) в недавней экспериментальной работе [7].

В [4] обсуждаются два основных механизма подавления током деформационных скачков. Первый из них связан с подавлением током процесса динамического деформационного старения (ДДС) дислокаций из-за изменения времени сегрегации примесных атомов на дислокациях, вызванных электромиграцией, а второй с растворением током малых преципитатов с меньшей электропроводностью по сравнению с матрицей, что может вызвать упрочнение сплава и подавление прерывистой деформации в рамках преципитатной модели эффекта ПЛШ в области инверсного поведения критической деформации появления первого скачка на кривых деформации. Цель настоящей работы состоит в исследовании с помощью комплекса высокоскоростных методов регистрации деформационных полос, измерения силового, акустического и теплового откликов влияния электрического тока на зарождение и распространение полос деформации, вызывающих прерывистую деформацию ПЛШ на примере алюминий-магниевого сплава АМг5.

Плоские холоднокатаные образцы промышленного сплава АМг5 (АА 5556$)$ (Al-5.35\%Mg-0.41\%Mn$0.25 \% \mathrm{Si}-0.27 \% \mathrm{Fe}, \mathrm{wt} . \%)$ в форме двусторонних лопаток с размерами рабочей части $0.5 \times 3 \times 6 \mathrm{~mm}$ деформировали с постоянной скоростью растяжения $\dot{\varepsilon}_{0}=3 \cdot 10^{-3} \mathrm{~s}^{-1}$ в жесткой испытательной машине (Instron, модель 3344). Методики регистрации деформационных полос на поверхности деформируемого образца, включающие обработку цифровых изображений, измерения акустической эмиссии (АЭ) и температуры, изложены в [5]. Отличительной особенностью настоящей работы является разработка и использование системы обратной связи между акустическим сигналом - предвестником деформационного скачка - и генератором прямоугольного импульса электрического тока, позволяющей пропускать импульсы тока заданной плотности и продолжительности на разных стадиях эволюции деформационных полос, связанных с зарождением, распространением и остановкой деформационных полос.

На рис. 1 представлена схема синхронизации различных стадий формирования деформационных полос с импульсами тока и системой воздушного охлаждения образца 1, которая включает акустический датчик 2, укрепленный на неподвижной лопатке образца, генератор импульсов тока 3, амплитудой 10-110 А, длительностью переднего фронта $1-10 \mu \mathrm{s}$ и длительностью крыши 0.01-3 s, компрессор 4 для воздушного охлаждения образца, блок управления 5 с системой контролируемой задержки между сигналом акустической эмиссии, вызванным начальной наиболее быстрой стадией развития деформационной полосы и генерацией прямоугольного импульса тока и одновременно запитыванием электромагнитного затвора 6 компрессора. Для синхронизации сигнала АЭ и/или сигнала тензодатчика, регистрирующе- 


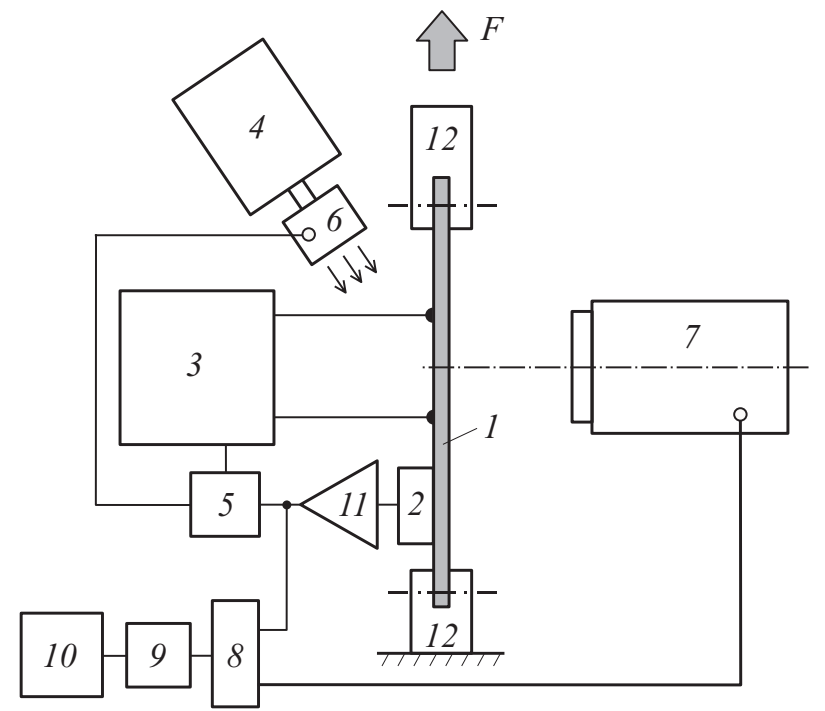

Pис. 1. Схема синхронизации акустического сигнала, связанного с зарождением первичной деформационной полосы, с генератором импульса тока, подавляющего полосообразование: 1 - образец, 2 - акустический датчик, 3 - генератор прямоугольных импульсов тока, 4 - компрессор, 5 - блок управления, включающий систему задержки генерации импульса тока, относительно начала генерации сигнала АЭ, 6 - электромагнитный затвор, 7 - скоростная видеокамера, 8 - коммутатор, 9 - АЦП, 10 - компьютер, 11 - предусилитель, 12 - стеклотекстолитовые захваты.

го силовой отклик системы машина-образец с кадрами видеосъемки, выходной сигнал высокоскоростной видеокамеры 7, представляющий собой последовательность прямоугольных импульсов (меандр) на частоте видеосъемки (500-2000 Hz), подавался вместе с сигналом АЭ (и/или тензодатчика) на коммутатор 8 и далее на аналого-цифровой преобразователь (АЦП) 9 и компьютер 10 .

Ранее в [5,6] было установлено, что оптимальные условия для подавления постоянным током прерывистой деформации ПЛШ в алюминий-магниевом сплаве с содержанием 5-6\% Mg соответствуют начальной стадии инверсного поведения критической деформации $\varepsilon_{c}$ в этих сплавах, а именно $\dot{\varepsilon}_{0}=(1-3) 10^{-3} \mathrm{~s}^{-1}$ и $T \approx 50-60^{\circ} \mathrm{C}$. В настоящей работе температура образца, контролируемая инфракрасным термометром Testo-845 c точностью $0.5^{\circ} \mathrm{C}$, поддерживалась равной $55^{\circ} \mathrm{C}$ с помощью климатической камеры. Одновременно с пропусканием импульса тока климатическая камера продувалась компрессором, что обеспечивало колебания температуры камеры в пределах от 50 до $60^{\circ} \mathrm{C}$ при длительности крыши импульса (и соответственно времени продува) до $3 \mathrm{~s}$. Отметим, что в сплаве АМг5 скачки напряжения при печном нагреве (в отсутствие тока через образец) исчезают при температурах выше $90^{\circ} \mathrm{C}$ [5]. Таким образом, разработанные методики позволяют комплексно исследовать in situ влияние импульсов тока на динамику деформационных полос, не связанное непосредственно с джоулевым нагревом.

Высокоскоростные исследования in situ динамики деформационных полос в сплавах системы $\mathrm{Al}-\mathrm{Mg}-\mathrm{Mn}[8,9]$ показали, что эволюция полосы деформации состоит из двух последовательных стадий: „быстрой“, продолжительностью до $10 \mathrm{~ms}$, на которой полоса зарождается от поверхностного источника на ребре плоского образца и пересекает сечение образца, и последующей „медленной“ стадии расширения полосы продолжительностью несколько сотен ms. Быстрая стадия развития деформационной полосы сопровождается скачком разгрузки амплитудой $\Delta \sigma$ от 3 до $15 \mathrm{MPa} \mathrm{в} \mathrm{зависимости} \mathrm{от} \mathrm{уровня}$ деформирующего напряжения и характерным сигналом АЭ, а медленная - восстановлением напряжения до исходного (до скачка) значения.

В первой серии экспериментов генератор прямоугольных импульсов тока запускали от начального всплеска сигнала АЭ длительностью $1 \mathrm{~ms}$, вызванного зарождением деформационной полосы. Время задержки $t_{d}$ между моментом зарождения полосы и началом генерации импульса тока устанавливали равным $1 \mathrm{~ms}$, длительность переднего фронта импульса тока $t_{f r}=10 \mu \mathrm{s}$, а длительность крыши $\tau=0.8 \mathrm{~s}$ устанавливали несколько меньшей среднего времени между скачками разгрузки $\overline{\Delta t} \approx 1.0 \mathrm{~s}$ (при $\dot{\varepsilon}_{0}=3 \cdot 10^{-3} \mathrm{~s}^{-1}, \sigma \approx 240-270 \mathrm{MPa}$, $T=55^{\circ} \mathrm{C}$ ). При такой схеме эксперимента момент включения тока приходится на начальную, быструю стадию развития деформационной полосы спустя не более $2 \mathrm{~ms}$ после ее зарождения. Амплитуду плотности тока устанавливали $j_{m}=60 \mathrm{~A} / \mathrm{mm}^{2}$, равной плотности постоянного тока, полностью подавляющего скачки напряжения при данных температурно-скоростных условиях деформирования [5].

Синхронные записи скачка разгрузки $\Delta \sigma$, сигнала АЭ, импульса тока и временной зависимости площади $A$ полосы - площади, заключенной между границами полосы, показали, что во время действия тока динамика полосы деформации не демонстрирует каких-либо особенностей, отличающих ее от динамики полосы в отсутствие тока [10]. Не изменяются также характер развития последующей медленной стадии эволюции полосы, амплитуда и форма скачка разгрузки. Таким образом, если постоянный ток плотностью $j_{m}=60 \mathrm{~A} / \mathrm{mm}^{2}$ включается сразу (в пределах $2 \mathrm{~ms}$ ) после зарождения полосы деформации, то он не оказывает заметного влияния на эволюцию этой полосы и характеристики скачка напряжения, вызванного развитием данной полосы деформации. Поэтому причину эффекта подавления током прерывистой деформации следует искать во влиянии тока на механизм зарождения деформационных полос.

Для экспериментального изучения этого вопроса необходимо включать ток за некоторое время $t_{t r}$ до ожидаемого скачка напряжения и измерять вероятность зарождения полосы деформации в зависимости от времени $t_{t r}$, которое имеет смысл времени предварительной электротоковой обработки металла до ожидаемо- 

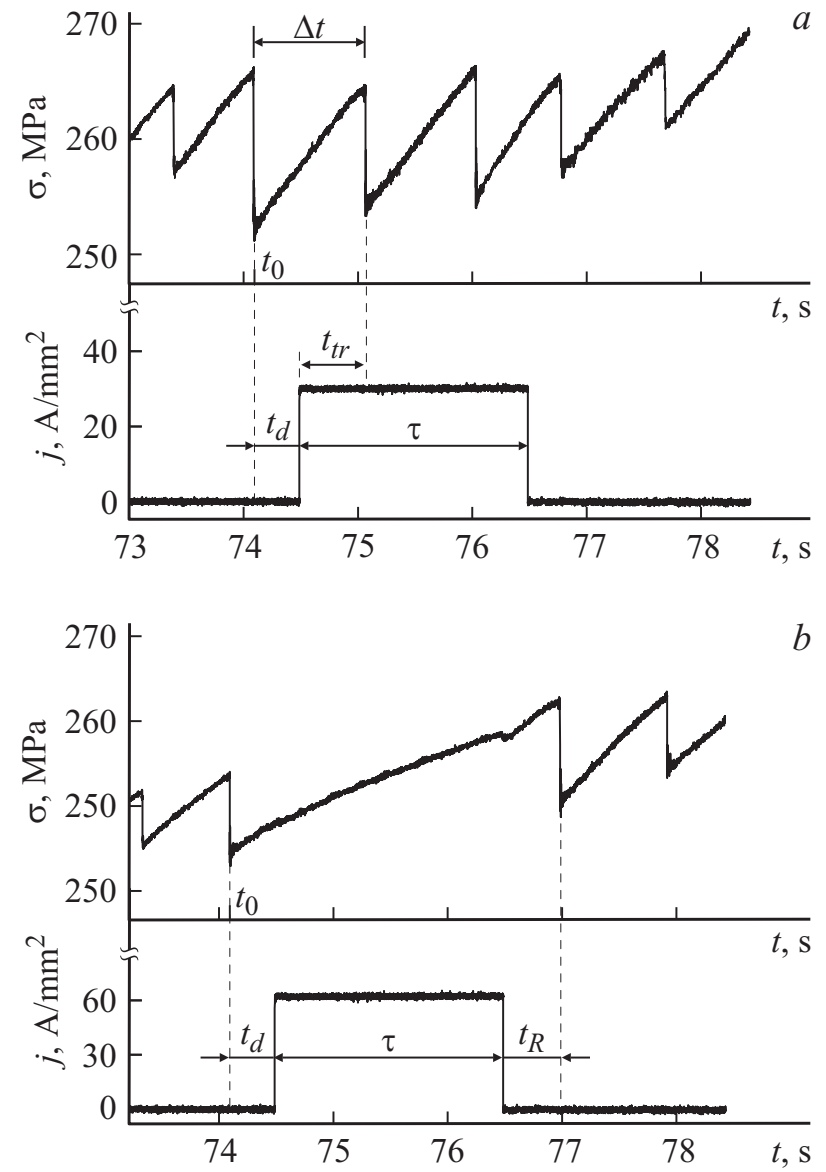

Рис. 2. Фрагмент скачкообразной кривой деформации сплава AMg5 и эпюра прямоугольного импульса тока без эффекта подавления $(a)$, когда $j_{m}<60 \mathrm{~A} / \mathrm{mm}^{2}$ и с подавлением скачков током $(b)$ при $j_{m}=60 \mathrm{~A} / \mathrm{mm}^{2} ; \Delta t-$ время между соседними скачками, $t_{d}<\Delta t-$ время задержки между скачком разгрузки и начальным моментом генерации импульса тока, $\tau_{R}-$ время восстановления скачков на деформационной кривой, $\tau=2 \mathrm{~s}-$ длительность „крыши“ прямоугольного импульса тока.

го момента зарождения полосы. Схема второй серии экспериментов состоит в следующем. На прерывистом участке кривой деформации в отсутствие электротокового воздействия выбирается фрагмент, содержащий 10-15 скачков напряжения, и измеряется среднее время между скачками $\overline{\Delta t}$. При заданных температурноскоростных условиях эксперимента $\left(\dot{\varepsilon}_{0}=3 \cdot 10^{-3} \mathrm{~s}^{-1}\right.$, $T \approx 55^{\circ} \mathrm{C}$ ) это время меняется от $\approx 1.0$ до $\approx 1.5 \mathrm{~s} \mathrm{c}$ ростом деформирующего напряжения. Генератор импульса запускается либо от акустического сигнала, либо сигнала тензодатчика по достижению величины всплеска сигнала АЭ или амплитуды скачка разгрузки некоторого порогового значения. Поскольку амплитуды сигналов АЭ и скачков разгрузки растут с ростом деформирующего напряжения, то варьированием порогового значения сигнала можно контролировать стадию деформирования, в которой генератор прямоугольного импульса тока включается от некоторого $i$-го скачка, амплитуда ко- торого достигла порога запуска генератора. Длительность крыши устанавливали приблизительно равной или больше среднего времени между скачками, т.е. $\tau \geq \overline{\Delta t}$ для исследования возможности этим импульсом тока подавить следующий $(i+1)$-й скачок деформации или несколько последующих скачков, а время задержки $t_{d}$ между моментом запуска генератора $t_{0}$ и началом генерации прямоугольного импульса тока варьировали от эксперимента к эксперименту в пределах от 0 до $t_{d} \approx \overline{\Delta t}$. Время предварительной электротоковой обработки вычисляется как $t_{t r}=\overline{\Delta t}-t_{d}$ (рис. 2). Таким образом, варьированием времени задержки $t_{d}$ данная методика позволяет контролировать время электротоковой обработки $t_{t r}$ и измерять вероятность подавления очередного $(i+1)$-ого скачка и соответственно подавлять процесс зарождения первичной полосы деформации.

Статистический анализ попыток подавления очередного скачка прямоугольным импульсом тока амплитудой $j_{m}=60 \mathrm{~A} / \mathrm{mm}^{2}$ показал, что при $0<t_{t r}<0.3 \mathrm{~s}$ вероятность подавления скачка напряжения равна нулю, при $t_{t r}>0.8 \mathrm{~s}$ эта вероятность близка единице, а в промежуточной области $0.3<t_{t r}<0.8 \mathrm{~s}$ вероятность подавления монотонно возрастает от нуля и стремится к единице. После окончания прямоугольного импульса тока скачки напряжения возобновляются через время $\tau_{R} \approx 0.5 \mathrm{~s}$, которое характеризует инерционность эффекта подавления током прерывистой деформации. Следовательно, для подавления процесса зарождения деформационных полос, а следовательно скачков напряжения, необходима предварительная обработка данного сплава в течение не менее $0.8 \mathrm{~s}$ током плотностью не менее $60 \mathrm{~A} / \mathrm{mm}^{2}$ (при $\dot{\varepsilon}_{0}=3 \cdot 10^{-3} \mathrm{~s}^{-1}, \quad T \approx 55^{\circ} \mathrm{C}$ ). Предположительно это время необходимо для растворения током малых преципитатов (зон Гинье-Престона), образующихся на ранних стадиях старения сплава. Полученные результаты подтверждают преципитатную модель подавления током прерывистой деформации ПЛШ [4].

Таким образом, экспериментально установлено, что электрический ток: а) подавляет процесс зарождения деформационных полос; б) не влияет на распространение полос, если зарождение произошло до включения тока. Механизмы распространения полос до сих пор являются дискуссионными вопросами $[10,12]$, в то же время о природе зарождения полос фактически ничего не известно. Из результатов настоящей работы следует, что электрический ток является примером селективного воздействия на процесс зарождения деформационных полос и может быть использован для его изучения.

Работа выполнена при финансовой поддержке Российского научного фонда (проект № 15-12-00035).

\section{Список литературы}

[1] Троицкий O.A. // Письма в ЖЭТФ. 1969. Т. 10. С. 18.

[2] Спицын В.И., Троицкий О.А. Электропластическая деформация металлов. М.: Наука, 1985. 161 с. 
[3] Трочикий О.А., Баранов Ю.В., Авраамов Ю.С., Шляпин А.Д. Физические основы и технологии обработки современных материалов: Теория, технология, структура и свойства: В 2 т.: Т. 1. Ижевск: Институт компьютерных исследований, 2004. 592 с.

[4] Шибков А.А., Золотов А.Е., Желтов М.А. и др. // Кристаллография. 2015. Т. 60. С. 929.

[5] Шибков А.А., Денисов А.А., Желтов М.А. и др. // ФТТ. 2015. T. 57. C. 228.

[6] Shibkov A.A., Denisov A.A., Zheltov M.A. et al. // Mater. Sci. Eng. A. 2014. Vol. 610. P. 338.

[7] Zhao K., Fan R., Wang L. // J. Mater. Eng. Perform. 2016. Vol. 25. N 3. P. 781.

[8] Шибков А.А., Золотов А.Е., Михлик Д.В. и др. // Деформация и разрушение материалов. 2009. № 9. С. 22.

[9] Шибков А.А., Золотов А.Е., Желтов М.А. // Изв. РАН. Сер. физ. 2012. Т. 76. № 1. С. 97.

[10] Шибков А.А., Золотов А.Е., Желтов М.А. // ФТТ. 2010. T. 52. Вып. 11. С. 2223.

[11] Hahner P. // Scripta Metall. Mater. 1993. V. 29. N 9. P. 1171.

[12] Hahner P., Ziegenbein A., Rizzi E., Neuhauser H. // Phys. Rev. B. 2002. Vol. 65. N 13. P. 134109. 